\title{
PLANIFICACIÓN DE LA PRODUCIÓN BASADA EN CONTROL PREDICTIVO PARA PLANTAS TERMOSOLARES
}

\author{
M.J. Vasallo, J.M. Bravo, E.J. Cojocaru y M.E. Gegúndez \\ Departamento de Ingeniería Electrónica, de Sistemas Informáticos y Automática, Universidad de Huelva \\ emails: manuel.vasallo@diesia.uhu.es, caro@uhu.es, emiliangelu.cojocaru@alu.uhu.es, gegundez@uhu.es
}

\section{Resumen}

Se presenta una estrategia basada en control predictivo para resolver la planificación de la producción en plantas termosolares con almacenamiento térmico participando en el mercado diario de electricidad. Mediante esta estrategia, la producción de electricidad es actualizada regularmente haciendo uso del estado actual de la planta y de las más recientes predicciones para el precio de la energía y el recurso solar. La estrategia propuesta se aplica, en un contexto de simulación, a una planta basada en colectores cilindro-parabólicos de $50 \mathrm{MW}$ con almacenamiento térmico bajo las hipótesis de predicción perfecta de precios y participación en el mercado diario español. Se ha analizado un caso de estudio basado en un periodo de cuatro meses con el propósito de abarcar una gran variedad de condiciones meteorológicas. Se han empleado valores reales para los precios de la energía, los costes de penalización, el recurso solar y su predicción. Los resultados muestran una mejora económica significativa frente a la tradicional estrategia a un día vista.

Palabras clave: Operación óptima de sistemas de potencia. Auto-programación de la producción. Integración en el mercado de la energía. Control predictivo basado en modelo. Modelado y simulación de sistemas de potencia.

\section{INTRODUCCIÓN}

La energía solar térmica de concentración (CSP, concentrating solar power) es una prometedora tecnología que ha despertado mucho interés en países como España y Estados Unidos, donde su desarrollo ha sido promovido gracias a políticas de subsidios. El interés en la tecnología CSP se basa en su carácter semi-gestionable cuando disponen de almacenamiento térmico (TES, thermal energy storage) y/o sistemas de respaldo basados en combustibles fósiles. Esta característica facilita su participación en el mercado eléctrico gracias a, entre otros factores, su capacidad para trasladar la producción hacia las horas con precios más al- tos. Es posible, por tanto, plantear el problema de la planificación óptima de la producción (también llamada auto-programación óptima). En un mercado desregularizado, los propietarios de las plantas de potencia tienen por objetivo maximizar sus beneficios a partir de las ventas de energía. Por otra parte, deben ofrecer al mercado su programa diario de producción con antelación. En consecuencia, se necesitan predicciones de precios de la energía y meteorológicas (en caso de productores de energía renovable) para resolver el problema de optimización.

Uno de los primeros trabajos sobre operación óptima en plantas CSP es [11. Se utilizaron dos modelos, la herramienta SAM [2] y un modelo de optimización basado en programación lineal entera mixta (MILP, mixed integer linear programming). Otros ejemplos con planteamientos MILP pueden encontrarse en [12] and [6].

Como consecuencia de errores en la predicción de irradiancia directa normal (DNI, direct normal irradiance), los propietarios de plantas CSP corren el riesgo de sufrir penalizaciones económicas por desvíos respecto al programa comprometido con el mercado. Con objeto de reducir el mencionado riesgo, los autores de este trabajo propusieron en [13] una estrategia de control predictivo (MPC, model-based predictive control) basada en programación entera mixta (MIP). Esta estrategia fue usada para la reprogramación de la producción y para el desarrollo del programa para el día siguiente. El control MPC es una estrategia ampliamente extendida en la industria y en el mundo académico dada su habilidad para tratar con modelos dinámicos y restricciones complejas (ver [10, 4]).

Este trabajo se inspira en la siguiente cuestión: ¿cuál es el impacto económico producido al usar la estrategia MPC en la auto-programación de plantas CSP? En este sentido, se ha desarrollado y probado una versión económica de la estrategia a partir del enfoque MPC general presentado en [13. Esta versión se basa en definir una función de coste donde la información sobre precios y costes de penalización se ha añadido con objeto de establecer un seguimiento económicamente óptimo 
del programa comprometido. De esta forma, la estrategia MPC busca compensar, al menos parcialmente, la penalización por desvíos mediante el incremento de los ingresos. La estrategia propuesta se aplica, en un contexto de simulación, a una planta basada en colectores cilindro-parabólicos de $50 \mathrm{MW}$ con almacenamiento térmico bajo las hipótesis de predicción perfecta de precios y participación en el mercado diario español.

En la Sección 2 se describe de forma genérica la estrategia MPC propuesta. En la Sección 3 se describen el caso de estudio y sus resultados. Las conclusiones se recogen en la Sección 4.

\section{DESCRIPCIÓN GENERAL DE LA ESTRATEGIA MPC DESARROLLADA}

Se asume la participación en el mercado diario de electricidad y la imposibilidad del productor de influir en los precios (dado su pequeño volumen de producción). El objetivo de la estrategia MPC es doble: 1) el seguimiento periódico y conveniente desde el punto de vista económico del programa comprometido con el mercado y 2) el desarrollo del programa óptimo de producción para el día siguiente en el momento previsto para ello. Este doble objetivo requiere que la ventana deslizante del MPC esté compuesta de dos intervalos de tiempo (ver Fig. 1): el intervalo para el seguimiento del programa comprometido (IS) y el intervalo para la generación del nuevo programa (INP). La reprogramación llevada a cabo por la estrategia MPC tiene por objeto el seguimiento durante el intervalo IS del programa comprometido. Al mismo tiempo debe tenerse en cuenta la maximización de los ingresos futuros dentro del intervalo INP. En el instante previsto para ello, el perfil de producción obtenido para el intervalo INP es entregado al mercado como plan de producción para el próximo día. A continuación se definen algunas variables y parámetros relacionados con la ventana deslizante:

- $t(i)=i \Delta t_{w}$ donde $i=0,1, \ldots$ son los instantes de tiempo en los que el control MPC genera salidas. El instante inicial de la ventana deslizante cuando esta está en la posición $i$ es referido como $t(i)$. El caso $i=0$ se refiere al instante $0.0 \mathrm{~h}$ del día actual D. $\Delta t_{w}$ es el paso de actualización del control MPC.

- $t_{\text {schedule_del }}$ es la hora límite durante el día D a la que hay que entregar el programa de producción para el día D+1. Esta hora depende del mercado de cada país.

- $t_{\text {schedule_end }}$ es el instante final del plan de

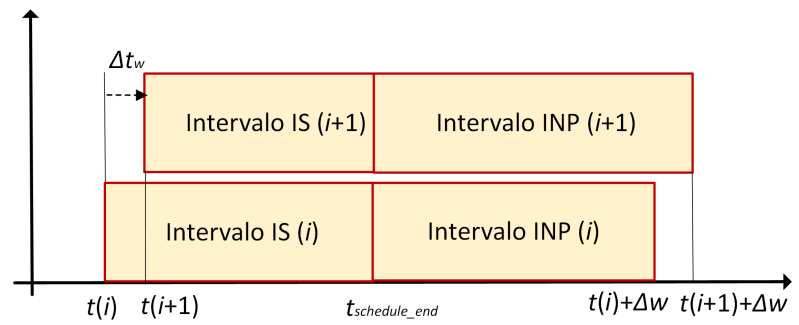

Figura 1: Ventana deslizante de la estrategia MPC

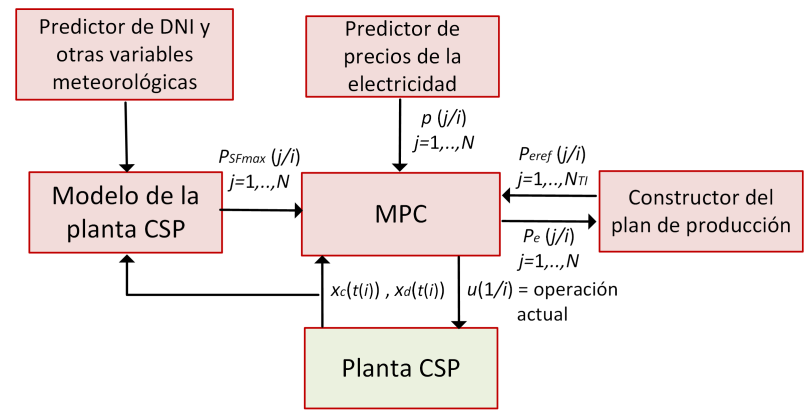

Figura 2: Diagrama de bloques de la estrategia $\mathrm{MPC}$

producción comprometido. Si el instante actual no supera $t_{\text {schedule_del }}, t_{\text {schedule_end }}$ es $24.0 \mathrm{~h}$ del día D. En caso contrario, $t_{\text {schedule_end }}$ es $24.0 \mathrm{~h}$ del día $\mathrm{D}+1$ porque el nuevo plan ya ha sido entregado.

- $\Delta w$ es la longitud de la ventana deslizante.

Los extremos de los intervalos IS e INP pueden observarse en la Fig. 1. en coherencia con las anteriores definiciones. Obsérvese que la longitud de la ventana deslizante es constante, no así la de ambos intervalos.

Cuando $t(i)=t_{\text {schedule_del }}$, el programa de producción resuelto por el control MPC para el intervalo INP hasta las $12.0 \mathrm{~h}$ del día $\mathrm{D}+1$ puede entregarse como plan de producción para ese día.

La Fig. 2 muestra el diagrama de bloques del control MPC. En cada posición $i$ de la ventana deslizante, el control MPC recibe la siguiente información:

1. El valor actual del estado continuo de la planta $\left(x_{c}(t(i))\right)$, por ejemplo el nivel de energía del almacenamiento y el estado térmico del campo solar (solar field, SF).

2. El valor actual del estado discreto de la planta $\left(x_{d}(t(i))\right)$, por ejemplo las fases de operación en SF, TES o turbina. 
3. Predicciones del precio de la energía realizadas el instante $t(i)(p(j / i)$, para $j=$ $1, \ldots, N)$, donde $j$ indica cada paso en el modelo MPC, $N=\Delta w / \Delta t_{o}$ es el número de pasos en la ventana deslizante y $\Delta t_{o}$ es el paso del modelo MPC expresado en horas.

4. Predicciones realizadas el instante $t(i)$ para el valor medio de la máxima potencia térmica disponible en SF $\left(P_{S F \max }(j / i)\right.$, para $j=$ $1, \ldots, N)$. El término 'máximo' se añade para indicar que un desenfoque parcial de los colectores del SF puede conllevar un decremento de la potencia disponible. Un modelo de la planta, predicciones para DNI y otras variables meteorológicas, y las condiciones iniciales $x_{c}(t(i))$ y $x_{d}(t(i))$ son elementos usados para generar estas predicciones.

5. Plan de producción comprometido aún por ejecutar $\left(P_{\text {eref }}(j / i)\right.$, para $\left.j=1, \ldots, N_{T I}\right)$, expresado en valores medios brutos de potencia eléctrica, donde $N_{T I}=\left(t_{\text {schedule_end }}-\right.$ $t(i)) / \Delta t_{o}$ es el número de pasos en el intervalo IS.

Como resultado de la optimización en la posición $i$, se generan las siguientes salidas:

1. Variables de decisión $(u(j / i)$, para $j=$ $1, \ldots, N)$. Sólo las variables de decisión $u(1 / i)$ se aplican en la planta como es habitual en el control MPC.

2. Valores medios brutos de la potencia eléctrica generada por la turbina calculada en el instante $t(i)\left(P_{e}(j / i)\right.$, para $\left.j=1, \ldots, N\right)$. Cuando $t(i)=t_{\text {schedule_del }}$, el nuevo plan de producción se construye con los valores dentro del intervalo INP hasta las 24.0h del día $\mathrm{D}+1\left(P_{\text {eref }}(j / i)=P_{e}(j / i)\right.$, para $j=N_{T I}+$ $1, \ldots, N_{T I}^{\prime}$, donde $N_{T I}^{\prime}=N_{T I}+24 / \Delta t_{o}$ es el nuevo número de pasos del intervalo INP).

La función objetivo a minimizar se expresa mediante la ecuación (1)

$$
\begin{aligned}
J(i)= & -\Delta t_{o} \sum_{j=1}^{N_{T I}}\left[p(j / i) P_{\text {enet }}(j / i)\right. \\
& \left.-\phi(j / i)\left(P_{\text {erefnet }}(j / i)-P_{\text {enet }}(j / i)\right)-f_{\text {cost }}(.)\right] \\
& -\Delta t_{o} \sum_{j=N_{T I}+1}^{N}\left[p(j / i) P_{\text {enet }}(j / i)-f_{\text {cost }}(.)\right] \\
& -s(E(N+1 / i))
\end{aligned}
$$

donde $\phi(j / i)$ es una estimación del coste de penalización por kWh de desviación durante la hora $j, P_{\text {enet }}(j / i)$ es la potencia eléctrica neta generada por la turbina, $P_{\text {erefnet }}(j / i)$ es la potencia eléctrica neta comprometida, $f_{\text {cost }}($.$) representan$ los costes de generación y $s(E(N+1 / i))$ es un elemento de valor terminal aplicado al nivel final de energía en TES. Nótese que la función $-J(i)$ representa la suma total de beneficios a lo largo de la ventana deslizante. En este trabajo se asume que la producción de electricidad no supera el valor comprometido. En consecuencia, el término $\phi(j / i)$ sólo hace referencia a desvíos negativos.

\section{CASO DE ESTUDIO}

En esta sección, la estregia MPC propuesta en este trabajo se aplica, en un contexto de simulación, a una planta basada en colectores cilindroparabólicos de $50 \mathrm{MW}$ con almacenamiento térmico. Además, dicha estrategia se compara con una estrategia de planificación a día vista (dayahead scheduling, DAS). La estrategia MPC usa el modelo MIP-MPC (ver Subsección 3.1), replanificación horaria, predicción solar a corto plazo actualizada cada hora y realimentación horaria del estado de la planta. La estrategia DAS se caracteriza por lo siguiente:

1. El plan para el día $D+1$ se genera el instante

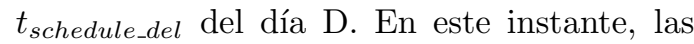
condiciones iniciales para el día $\mathrm{D}+1$ se estiman empleando el estado actual de la planta, la predicción a un día vista y el plan de producción comprometido aún po ejecutar.

2. El plan de producción es ejecutado sin ninguna replanificación. Es decir, la producción cada hora es el valor máximo posible que no supere el valor comprometido.

El modelo de optimización empleado por la estrategia DAS es referido en este trabajo como modelo MIP-DAS (ver Subsección 3.1). La tabla 1 resume toda la información sobre las estrategias de planificación estudiadas y sus modelos de optimización.

La planta CSP analizada en este caso de estudio se basa en el modelo presentado en [5, y también usado en [13, que describe la planta Andasol 2 en Granada, España. Algunas características de este modelo (adaptadas a este caso de estudio) pueden consultarse en la tabla 2 .

A continuación se describen las principales características del escenario de simulación desarrollado para este caso de estudio. Se ha considerado un periodo de tiempo suficientemente largo (desde 01/02/2013 hasta $31 / 05 / 2013$ ) con el propósito 
Tabla 1: Descripción de las estrategias de planifición analizadas

\begin{tabular}{ccccc}
\hline Estrat. & Modelo & Replanif. & $\begin{array}{c}\text { Predic. } \\
\text { a corto } \\
\text { plazo }\end{array}$ & $\begin{array}{c}\text { Realim. } \\
\text { del estado } \\
\text { de la planta }\end{array}$ \\
\hline MPC & MIP-MPC & Horaria & Horaria & Horaria \\
DAS & MIP-DAS & No & No & Diaria \\
\hline
\end{tabular}

Tabla 2: Características de la planta CSP

Capacidad bruta de turbina

Capacidad de SF

Capacidad térmica en modo sólo solar

Capacidad térmica en modo sólo TES

Múltiple solar

Capacidad de TES (modo sólo TES)

Eficiencia de turbina (plena carga)

Sin generación eléctrica con calderas
$52.5 \mathrm{MW}-\mathrm{e}$

$250 \mathrm{MW}-\mathrm{t}$

$140 \mathrm{MW}-\mathrm{t}$

$119 \mathrm{MW}-\mathrm{t}$

1.8

8 hours

$38 \%$

de testear una gran variedad de condiciones meteorológicas. Se asume la participación en el mercado diario de electricidad, la imposibilidad del productor de influir en los precios (dado su pequeño volumen de producción) y predicción perfecta para los precios de la energía (obtenidos de la web del operador del mercado ibérico OMIE 1]). No se considera ninguna prima para la producción renovable. Los costes de penalización por MWh desviado respecto al plan comprometido son obtenidos del operador el sistema (Red Eléctrica de España). Por otra parte, los costes de generación se han supuesto despreciables por simplicidad. Las resoluciones del plan de producción y del modelo MIP-MPC son horarias. La frecuencia de replanificación es también horaria. Por tanto, $\Delta t_{o}=\Delta t_{w}=1.0 \mathrm{~h}$ (ver Fig. 1). El parámetro $t_{\text {schedule_del }}$ se considera igual a $10.0 \mathrm{~h}$ (mercado español en 2013). El parámetro $\Delta w$ se fija a $48 \mathrm{~h}$. En consecuencia, la duración del intervalo INP a las $10.0 \mathrm{~h}$ es $34 \mathrm{~h}$, es decir, un esquema intermedio entre las planificaciones a un día vista y a dos días vista 14. Para generar las predicciones $P_{S F \max }(j / i)$ (ver Fig 2 ), se usa el modelo detallado en [13. El DNI y la temperatura ambiente son las únicas variables meteorológicas consideradas y la predicción de temperatura ambiente se supone perfecta y se crea mediante datos TMY2 3. La planta CSP se representa mediante un modelo de resolución horaria para evitar un alto tiempo de simulación, al igual que en [7, 8]. Particularmente, se emplea un modelo MIP derivado del propio modelo MIP-MPC. Por lo tanto, las diferencias de producción sólo se deben a errores de predicción del DNI. Este modelo se denomina en este trabajo modelo MIPplant. Se dispone de un conjunto de predicciones a un día vista de radiación solar obtenidas por el modelo Integrated Forecast System (IFS) del Centro Europeo de Predicciones Meteorológicas a

Mediano Plazo (ECMWF). Además, también está disponible un conjunto de medidas de radiación solar. Ambos conjuntos se convierten a potencia térmica máxima disponible en SF mediante simulación con el modelo detallado de la planta CSP. En este estudio también se tienen en cuenta las predicciones a corto plazo de DNI. Se pueden consultar varios métodos en [9]. En ausencia de datos de predicción a corto plazo, se ha desarrollado un predictor sintético, que trabaja directamente con la variable $P_{S F \max }(j / i)$ para evitar la conversión de valores DNI. La ecuación (2) describe el predictor sintético a corto plazo utilizado.

$$
\begin{gathered}
P_{S F m a x \_S T F}(k / i)=P_{S F m a x \_a c t u a l}(k / i)+ \\
+r(k)\left(P_{S F m a x \_D A}(k / i)-P_{S F m a x \_a c t u a l}(k / i)\right)
\end{gathered}
$$

para $k=1$ to $N_{S T F}+1$; donde $N_{S T F}$ es el horizonte (horas) de la predicción a corto plazo; $r(k)$ es una función lineal con el índice $k, r(1)=0 ; r\left(N_{S T F}+\right.$ $1)=1 ; \quad P_{S F m a x \_S T F}(k / i), \quad P_{S F m a x \_a c t u a l}(k / i)$ y $P_{S F m a x \_D A}(k / i)$ son las máximas potencias térmicas disponibles en $\mathrm{SF}$ predichas a corto plazo, real y predichas a un día vista, respectivamente. Obsérvese que el porcentaje de error en la predicción a un día vista añadido al valor real para generar la predicción a corto plazo de la primera hora es 0 . Este porcentaje crece linealmente con el tiempo hasta llegar a $100 \%$ cuando $k=N_{S T F}+1$, es decir, fuera del horizonte de predicción a corto plazo. Valores típicos para $N_{S T F}$ son 5 o 6 horas [9, 8.

Los modelos MIP se describen en la Subsección 3.1. La Subsección 3.2 presenta las propiedades de los siguientes datos de entrada: recurso solar, su predicción a un día vista y coste de penalización por kWh de desviación, Finalmente, los resultados y las conclusiones se exponen en la Subsection 3.3 .

\subsection{MODELOS MIP}

En esta subsección se describen los tres modelos MIP, todos de resolución horaria. Los modelos MIP-DAS y MIP-plant se derivan del modelo MIP-MPC. La formulación de los tres modelos MIP para este caso de estudio no conllevó el empleo de elementos no lineales, excepto las variables binarias. Por lo tanto, son modelos MILP.

Es importante hacer una aclaración en este momento: se supone que el operador y los sistemas de control de la planta toman decisiones basadas en dos objetivos con diferente prioridad [13]. El objetivo de alta prioridad es minimizar el error de producción. Una vez que se cumpla este objetivo, la prioridad es minimizar la potencia térmica desenfocada en SF. Por lo tanto, la planta en estudio 
tiene una única variable de decisión independiente en relación con el reparto de potencia, por ejemplo, la consigna para la generación de electricidad. Por tanto, la acción del MPC $u(1 / i)=P_{e_{-} S P}(1 / i)$. Se usa la ecuación $P_{e_{-} S P}(1 / i)=P_{e}(1 / i)$ para obtener la consigna, donde $P_{e}(1 / i)$ es un valor generado por el control MPC.

\subsubsection{Modelo MIP-MPC.}

El conjunto de ecuaciones y desigualdades que compone el modelo MIP-MPC, junto con los valores de los parámetros presentes, se puede consultar en [13]. La función objetivo a minimizar se muestra en la expresión (3), que es un caso específico de la función objetivo en la Sección 2.

Minimize

$$
\begin{gathered}
J(i)=\quad-\Delta t_{o} \sum_{j=1}^{N_{T I}}\left[p(j / i) P_{\text {enet }}(j / i)\right. \\
\left.-\phi(j / i)\left(P_{\text {erefnet }}(j / i)-P_{\text {enet }}(j / i)\right)\right] \\
-\Delta t_{o} \sum_{j=N_{T I}+1}^{N}\left(p(j / i) P_{\text {enet }}(j / i)\right) \\
-K E(N+1 / i)
\end{gathered}
$$

En esta función objetivo, no se tienen en cuenta los costes de generación y $K E(N+1 / i)$ es el elemento de valor terminal, formado por un valor proporcional al nivel final de energía en TES, con la constante $K$ definida por la ecuación $K=\eta p_{\text {vlow }}$, donde $\eta$ es un factor de eficiencia para convertir energía almacenada en energía eléctrica neta y $P_{\text {vlow }}$ es un valor muy inferior al precio mínimo de la electricidad durante el periodo simulado. De esta manera, el elemento de valor terminal hace que la energía térmica desenfocada sea tan baja como sea posible una vez se han maximizado los beneficios económicos (sin tener en cuenta el elemento de valor terminal). En el mercado español, la desviación sobre la producción comprometida, en caso de que requiere la intervención del operador del sistema, genera costes de penalización. Estas sanciones están asociadas con los costos incurridos para estabilizar el sistema, y no siguen ninguna función preestablecida. Por lo tanto, estos costes son difíciles de estimar. Un valor promedio de $\phi(j / i)$ se supone en la subsección 3.2 .

\subsubsection{Modelo MIP-DAS.}

El modelo MIP-DAS es un modelo de optimización que genera el plan de producción para el día $\mathrm{D}+1$ cuando $t(i)=t_{\text {schedule_del }}$ durante el día D cuando la estrategia DAS se está ejecutando. El modelo MIP-DAS se deriva del modelo MIP-MPC de la siguiente manera: el intervalo IS se elimina y el intervalo INP comienza la hora 0 del día $\mathrm{D}+1$. Los valores iniciales para el instante previo a la hora 0 del día $\mathrm{D}+1$ se estiman en $t_{\text {schedule_del }}$ del día $D$ utilizando el estado actual de la planta, la predicción a un día vista y el plan comprometido aún por cumplir.

\subsubsection{Modelo MIP-plant.}

Para evitar un tiempo de computación elevado, la representación de la planta CSP se realiza mediante un modelo MIP de resolución horaria. Se compone de dos modelos consecutivos de optimización derivados del modelo MIP-MPC. Los dos objetivos con diferente prioridad que guían las decisiones del operador de la planta y los sistemas de control (ver el comienzo de la sección 3.1) explican este esquema. Este modelo recibe cada hora la consigna generada a partir de las estrategias MPC o DAS. A continuación, se obtiene la evolución de la generación eléctrica y el estado de la planta en base a esta actualización horaria del punto de consigna, el valor real del recurso solar y los objetivos mencionados anteriormente.

\subsection{DESCRIPCIÓN DE LOS DATOS DE ENTRADA}

En esta subsección se describen las características del recurso solar, su predicción a un día vista y los costos de penalización por MWh de desviación para el período de tiempo estudiado. La Fig. 3 muestra los valores promedio horarios de la potencia térmica máxima disponible en SF, $P_{S F m a x ~ a c t u a l}(j)$, que se han obtenido usando datos de radiación solar y el modelo detallado de la planta CSP. Como puede observarse en la figura, a medida que avanzan los días, el perfil de $P_{S F m a x \_a c t u a l}(j)$ aumenta en intensidad y longitud. Además, aproximadamente los primeros setenta días presentan una alta variabilidad meteorológica, mientras que la estabilidad aumenta durante los últimos cincuenta días.

Una variable que puede influir en el desempeño de las estrategias de planificación es, evidentemente, el error de predicción de la máxima potencia térmica disponible en SF. En general, el error de predicción aumenta con la variabilidad meteorológica, es decir, los días de invierno presentan errores de predicción más altos que los días claros de verano. Con el fin de caracterizar el error de predicción a un día vista, la tabla 3 muestra algunas métricas a escala mensual, donde se han excluido las horas nocturnas. La media de la máxima potencia térmica disponible en SF se denomina $\bar{P}_{S F m a x \_a c t u a l}$. El error absoluto medio relativo y el error de sesgo medio relativo se denotan por $r M A E$ y $r M B E$ respectivamente, (vea [7] para las expresiones de estas métricas). Algunas comentarios pueden realizarse. La media de la máxima potencia térmica disponible en SF 


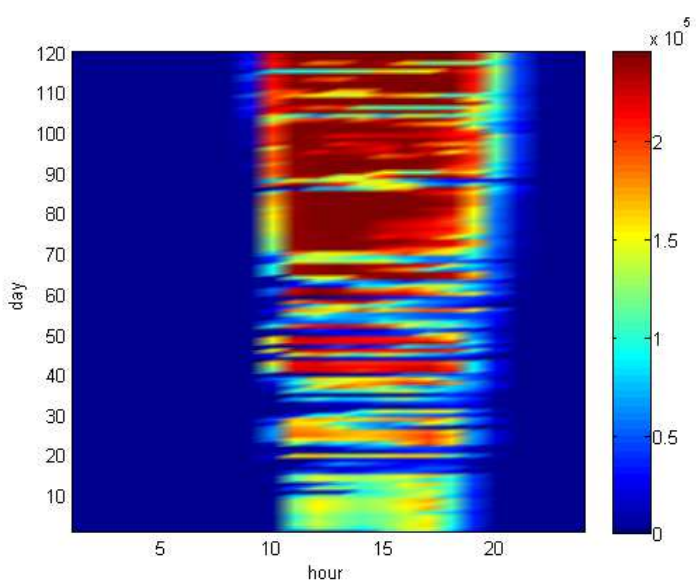

Figura 3: Valores medios horarios de la máxima potencia térmica disponible en $\mathrm{SF}(\mathrm{kW})$

Tabla 3: Métricas de la predicción a un día vista para la máxima potencia térmica disponible en SF

\begin{tabular}{cccc}
\hline Mes & $\bar{P}_{S F m a x \_a c t u a l}(\mathrm{MW})$ & $r M A E(\%)$ & $r M B E(\%)$ \\
\hline Feb. & 82.5 & 32.1 & 3.8 \\
Mar. & 87.6 & 52.8 & 8.4 \\
Abr. & 152.4 & 25.7 & -5.3 \\
May. & 164.4 & 17.8 & 10.6 \\
\hline
\end{tabular}

aumenta cerca del $100 \%$ de Febrero a Mayo. El error relativo es mayor en los meses de invierno. De hecho, Marzo ha sido particularmente malo en el período estudiado. Finalmente, el error de sesgo puede variar ampliamente.

La estrategia MPC propuesta emplea un valor $\phi(j, i)$ constante. Concretamente, $\phi(j, i)=\bar{\phi}$, donde $\bar{\phi}=7.69$ Euros/MWh es el valor medio de los costes de penalización durante el primer periodo de seis meses de 2013.

\subsection{RESULTADOS Y DISCUSIÓN}

En esta subsección se muestran y comentan los resultados de la simulación. Es importante remarcar que hay muchos factores que afectan los resultados económicos de las estrategias de planificación, por ejemplo, las normas regulatorias del mercado de la electricidad, el clima local de la planta, las predicciones, el diseño de la planta, las hipótesis de simplificación aplicadas y los modelos utilizados 8. Por tanto, las conclusiones extraídas de este caso de estudio podrían ser diferentes en otros escenarios. A continuación, se realizan los siguiente análisis: (1) comparación económica entre las estrategias MPC y DAS; (2) cálculo mensual del porcentaje de mejora en los beneficios de la estrategia MPC respecto a la estrategia de DAS; y (3) análisis energético de ambas estrategias.

La tabla 4 muestra los resultados económicos totales de las estrategias DAS y MPC, donde puede
Tabla 4: Resultados económicos de las estrategias DAS y MPC

\begin{tabular}{|c|c|c|}
\hline & DAS & MPC \\
\hline Ingresos (Euros) & 2148321 & 2228481 \\
\hline Penalizaciones (Euros) & 38969 & 58949 \\
\hline Beneficios (Euros) & 2109352 & 2169532 \\
\hline
\end{tabular}

Tabla 5: Porcentaje de mejora en los beneficios de la estrategia MPC respecto a la estrategia DAS

\begin{tabular}{|c|c|c|c|}
\hline Febrero & Marzo & Abril & Mayo \\
\hline $4.4 \%$ & $7.3 \%$ & $3.4 \%$ & $0.9 \%$ \\
\hline
\end{tabular}

apreciarse la mejora de la estrategia MPC en relación a los beneficios finales.

La tabla 5 muestra, para cada mes, el porcentaje de mejora en los beneficios de la estrategia MPC con respecto a la estrategia DAS. Como puede observarse, la estrategia MPC obtiene mejoras sustanciales cuando la inestabilidad meteorológica está presente. De hecho, el mejor resultado se obtiene en marzo, es decir, el mes con peor predicción para la máxima potencia térmica disponible en SF. En este sentido, la estrategia MPC puede compensar situaciones de baja calidad en la predicción.

La tabla 6 muestra algunos resultados energéticos de las estrategias DAS y MPC. Se observa que la generación es ligeramente inferior cuando se ejecuta la estrategia MPC. Además, los valores asociados con la estrategia MPC para la desviación y la energía desenfocada son también peores. La mejora económica de la estrategia MPC queda reflejada en el parámetro Precio de venta equivalente. Este parámetro se define como la relación entre los beneficios totales y la generación total. Los peores resultados energéticos de la estrategia MPC se explican por su capacidad para admitir desviaciones con objeto de poder reservar energía para posibles mayores ingresos futuros, como su mayor nivel medio de energía en TES confirma. Esta capacidad se basa en el deslizamiento horario de la ventana MPC, que va incorporando nueva información (precisa o no). En cualquier caso, aunque la desviación total puede ser mayor con la estrategia MPC, esta estrategia la distribuye aprovechando las horas más favorables obteniendo una mejora de $2,33 \%$ en el precio de venta equivalente. La Fig. 4 muestra la distribución de la generación eléctrica en relación con intervalos de precios para ambas estrategias. Se puede observar el desplazamiento de la generación de la estrategia MPC hacia precios altos en comparación con la estrategia DAS.

Las siguientes conclusiones pueden extraerse de- 
Tabla 6: Resultados energéticos de las estrategias DAS y MPC

\begin{tabular}{cccc}
\hline Valor medio horario & DAS & MPC & $\%$ \\
\hline Generación (MWh-e) & 12.42 & 12.37 & $-0.43 \%$ \\
Desviación (MWh-e) & 1.46 & 1.76 & $20.68 \%$ \\
Energía desenf. (MWh-t) & 4.26 & 4.39 & $3 \%$ \\
Nivel de energía en TES (\%) & 23.29 & 28.04 & $20.37 \%$ \\
\hline Prec. venta equiv. (Euros/MWh-e) & 64 & 65.49 & $2.33 \%$ \\
\hline
\end{tabular}

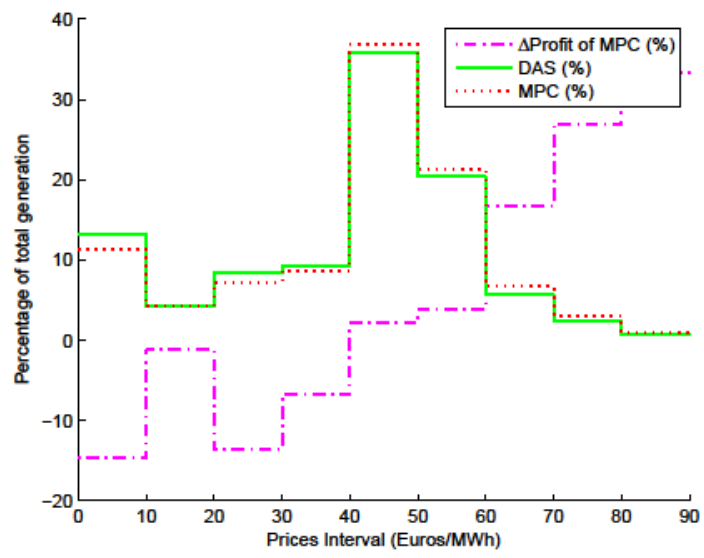

Figura 4: Distribución de la generación respecto a intervalos de precios para las estrategias DAS y MPC (\%)

spués de analizar los resultados:

1. La estrategia MPC obtiene mayores beneficios totales que la estrategia DAS durante el período de cuatro meses.

2. La mejora en los beneficios de la estrategia MPC en relación con la estrategia DAS es mayor en períodos con baja calidad en la predicción del recurso solar. En este sentido, el porcentaje de mejora durante Marzo es superior al $7 \%$.

3. La mejora de la estrategia MPC se basa en la replanificación horaria, que se adapta a la situación actual y desplaza la generación a horas de alto precio.

En opinión de los autores, la estrategia MPC propuesta podría alcanzar mejores resultados en las siguientes situaciones:

1. En el caso real de predicción imperfecta del precio de la energía, la estrategia MPC podría superar más claramente a la estrategia DAS gracias al conocimiento perfecto de los precios del día actual a partir de una hora determinada.

2. En escenarios con un mayor nivel de costes de penalización, el desempeño de la estrategia MPC puede tener más importancia.
3. Se pueden evaluar modelos más complejos para el término de penalización en la función de optimización.

4. Otros aspectos como analizar el efecto de la longitud de la ventana deslizante, o incluir términos robustos en el problema de optimización, son interesantes para investigar.

\section{CONCLUSIONES}

En este trabajo se ha propuesto una estrategia MPC para abordar la planificación óptima de producción en plantas CSP con TES. Uno de los principales obstáculos presente en esta clase de problemas es el coste de penalización que aplica el mercado cuando se produce una desviación respecto al plan de producción comprometido. La existencia de este desvío se debe principalmente a la predicción limitada del recurso solar. El enfoque propuesto aborda este problema mediante dos actuaciones: (1) la replanificación periódica de la producción en busca de una solución más ventajosa económicamente haciendo uso de información actualizada de la predicción solar y el estado de la planta, y (2) el desarrollo, en el instante previsto para ello, de un plan de producción para el día siguiente más fácil de ejecutar gracias al uso de una mejor estimación para las condiciones iniciales del día siguiente. Para realizar las actuaciones anteriores, la función objetiva del MPC se construye con términos económicos en los que se emplean precios predichos de la electricidad y estimaciones de los costes de penalización. La estrategia propuesta se ha aplicado, en un contexto de simulación, a una planta CSP de 50 MW basada en colectores cilindro-parabólicos con almacenamiento térmico bajo las hipótesis de participación en el mercado diario español de la electricidad y una perfecta predicción de precios. Este caso de estudio se basa en un período de cuatro meses para probar varias condiciones meteorológicas. El enfoque propuesto se ha comparado con una estrategia de referencia basada en una planificación a día vista. El análisis comparativo abarca resultados económicos y energéticos. Se observa una mejora económica significativa, especialmente en períodos con malas predicciones de recurso solar. Finalmente, se han indicado varias líneas de investigación futuras: 1) análisis de escenarios con predicciones imperfectas de los precios de la electricidad o niveles más altos de penalización, 2) desarrollo de métodos más complejos para estimar el costo de la penalización, y 3) provisión de robustez para el enfoque propuesto.

\section{Agradecimientos}

Este trabajo ha sido financiado por el proyecto 
DPI2016-76493-C3-2-R del Ministerio de Economia y Competitividad (España).

\section{Referencias}

[1] OMIE. Last access: 28.04.17. http://www . omie.es/, 2017.

[2] The SAM website. Last access: 28.04.17. https://sam.nrel.gov/, 2017.

[3] User's manual for TMY2s. Last access: 28.04.17. http://rredc.nrel.gov/solar/ pubs/tmy2/, 2017.

[4] José Mamuel Bravo, T. Alamo, and E. F. Camacho. Robust MPC of constrained discrete-time nonlinear systems based on approximated reachable sets. Automatica, 42(10):1745 - 1751, 2006.

[5] Isabel Llorente Garcia, Jose Luis Alvarez, and Daniel Blanco. Performance model for parabolic trough solar thermal power plants with thermal storage: Comparison to operating plant data. Solar Energy, 85(10):24432460, 2011.

[6] Christoph Kost, Christoph M. Flath, and Dominik Most. Concentrating solar power plant investment and operation decisions under different price and support mechanisms. Energy Policy, 61(0):238 - 248, 2013.

[7] Birk Kraas, Marion Schroedter-Homscheidt, and Reinhard Madlener. Economic merits of a state-of-the-art concentrating solar power forecasting system for participation in the Spanish electricity market. Solar Energy, 93(0):244 - 255, 2013.

[8] Edward W. Law, Merlinde Kay, and Robert A. Taylor. Calculating the financial value of a concentrated solar thermal plant operated using direct normal irradiance forecasts. Solar Energy, 125:267 - 281, 2016.

[9] Edward W. Law, Abhnil A. Prasad, Merlinde Kay, and Robert A. Taylor. Direct normal irradiance forecasting and its application to concentrated solar thermal output forecasting — A review. Solar Energy, 108(0):287 307, 2014.

[10] David Q. Mayne. Model predictive control: Recent developments and future promise. $\mathrm{Au}$ tomatica, 50(12):2967 - 2986, 2014.

[11] R. Sioshansi and P. Denholm. The value of concentrating solar power and thermal energy storage. Sustainable Energy, IEEE Transactions on, 1(3):173-183, Oct 2010.
[12] J. Usaola. Operation of concentrating solar power plants with storage in spot electricity markets. Renewable Power Generation, IET, 6(1):59-66, January 2012.

[13] Manuel Jesús Vasallo and José Manuel Bravo. A MPC approach for optimal generation scheduling in CSP plants. Applied Energy, 165:357 - 370, 2016.

[14] Michael Wittmann, Markus Eck, Robert Pitz-Paal, and Hans Muller-Steinhagen. Methodology for optimized operation strategies of solar thermal power plants with integrated heat storage. Solar Energy, 85(4):653 - 659, 2011. SolarPACES 2009. 